\title{
Transatlantica
}

Revue d'études américaines. American Studies Journal

Line Breaks in America: the Odds and Ends of Poetry

\section{Hervé Mayer, Guerre sauvage \& empire de la liberté}

\section{Sophie Gergaud}

\section{OpenEdition}

Journals

Édition électronique

URL : https://journals.openedition.org/transatlantica/16648

DOI : 10.4000/transatlantica. 16648

ISSN : $1765-2766$

\section{Éditeur}

Association française d'Etudes Américaines (AFEA)

\section{Référence électronique}

Sophie Gergaud, «Hervé Mayer, Guerre sauvage \& empire de la liberté », Transatlantica [En ligne], 1 | 2021, mis en ligne le 01 juin 2021, consulté le 02 février 2023. URL : http://journals.openedition.org/ transatlantica/16648; DOI : https://doi.org/10.4000/transatlantica.16648

Ce document a été généré automatiquement le 2 février 2023.

\section{(c)}

Creative Commons - Attribution - Pas d'Utilisation Commerciale - Pas de Modification 4.0 International - CC BY-NC-ND 4.0

https://creativecommons.org/licenses/by-nc-nd/4.0/ 


\title{
Hervé Mayer, Guerre sauvage \& empire de la liberté
}

\author{
Sophie Gergaud
}

\section{RÉFÉRENCE}

Hervé Mayer, Guerre sauvage \& empire de la liberté, Clermont-Ferrand : Presses

Universitaires Blaise Pascal, 2020, 324 pages, ISBN 978-2-8451-962-3, 26,50 euros.

1 En 2016, Hervé Mayer soutenait sa thèse en civilisation américaine et études filmiques sous le titre de « Guerre sauvage et empire de la liberté : prolongements du mythe de la Frontière dans le cinéma américain post-western ». Partant de la résurgence récente du mythe de la Frontière dans la rhétorique politique de la « guerre contre la terreur », il y démontrait que, loin d'avoir été marginalisé au sein de la culture étatsunienne, le mythe de la Frontière s'était en réalité diversifié, prolongé et consolidé. Son ouvrage Guerre sauvage \& empire de la liberté, publié en 2020, reprend cette analyse sociohistorique des représentations du mythe de la Frontière dans le cinéma populaire étatsunien. En s'appuyant sur le même corpus principal constitué de six films de genres génériques différents, sortis entre 1968 et 1986 - Délivrance de John Boorman (1972), L'inspecteur Harry de Don Siegel (1971), Platoon d'Oliver Stone (1986), La Planète des singes de Franklin Schaffner (1968), Easy Rider de Dennis Hopper (1969) et Star Wars IV: Un nouvel espoir de George Lucas (1977) -, il s'attache à éclairer la persévérance du mythe de la Frontière sur les plans narratifs et idéologiques, depuis la fin du XIXe siècle jusqu'à l'époque contemporaine, avec un point d'articulation au cours des années 1960, au moment où les critiques du mythe classique entraînent une diversification de ses représentations.

Rompant avec les lectures évolutionnistes classiques qui dominent les études du western filmique, l'auteur invite à juste titre à repérer, au-delà des crises traversées par le genre, les effets de continuité du mythe de la Frontière qui informe la production cinématographique étatsunienne dans son ensemble, que ce soit dans le cadre du road 
movie, du film policier ou de la science-fiction. Rappelant que «le mythe de la Frontière n'est pas le western et ne se réduit pas au récit de la conquête de l'Ouest ", il soutient qu'« une grande partie du cinéma étatsunien place la Frontière au cœur de ses préoccupations» et qu'elle "habite toujours la rhétorique politico-médiatique, la culture populaire et la langue étatsuniennes et garde une place de choix dans les mécanismes de construction identitaire des Etats-Unis contemporains» (11). L'auteur aborde ainsi de façon pertinente le mythe de la Frontière comme "un système signifiant structuré » (13) et montre qu'il se caractérise par « une fluidité des formes mais une permanence des structures » (14).

3 L'introduction est l'occasion de rappeler les concepts clés qui alimenteront ensuite largement la réflexion. Si l'auteur revient notamment «à la source» de la Frontière entendue par Turner comme « point de rencontre entre la civilisation et la sauvagerie " d'où émerge le nouvel homme étatsunien, il précise toutefois que cette définition initiale ne limite pas le concept dans l'espace ou le temps (17). Il esquisse ici les possibles incarnations de la Frontière que ce soit sur d'autres territoires ou à d'autres époques que l'Ouest historique. Le mythe est quant à lui compris comme une lecture possible du monde et l'auteur précise que le « fait qu'un mythe se modifie n'est pas tant un signe de crise que de vitalité » (20). C'est cette vision fluide du mythe qui lui permet ensuite d'appréhender les transformations subies par le mythe de la Frontière au cours des années 1960 comme une série d'ajustements idéologiques témoignant de son dynamisme davantage que de son déclin tant de fois annoncé. Enfin, après avoir rappelé les grandes caractéristiques des traditions majeures étatsuniennes, celle du nationalisme expansionniste couplé à l'impérialisme racialiste d'un côté, et celles de l'agrarianisme et du populisme de l'autre, l'auteur propose de suivre deux trajectoires principales qui relient mythe de la Frontière et cinéma contemporain: depuis la conquête de l'Ouest de Theodore Roosevelt jusqu'aux représentations contemporaines de la guerre sauvage tout d'abord, puis de la théorie de la Frontière de Frederick Turner jusqu'aux incarnations cinématographiques de l'empire de la liberté de l'autre. La structure du livre est donc logiquement organisée en deux parties qui reprennent cette articulation : la première partie intitulée « De John Wayne à John Rambo » étant dédiée à la guerre sauvage, tandis que la deuxième, « Renouer avec l'idéalisme de l'Ouest », est consacrée aux films incarnant l'empire de la liberté.

4 Le premier chapitre "Deliverance: la dégénération par la violence » explore les conséquences destructrices du mythe de la Frontière, cette dernière étant considérée comme la source d'une violence traumatique pour l'Amérique contemporaine. À travers l'exemple du film de John Boorman qui donne son titre au chapitre, l'auteur montre que l'ensauvagement des protagonistes ne mène pas à l'habituelle régénération de la civilisation, mais plutôt à la conscience coupable du meurtre fondateur et du prix du progrès. C'est là, selon lui, l'incarnation de ce que Richard Slotkin a qualifié de «turnerisme inversé »: la Frontière est toujours à l'origine du caractère étatsunien, mais celui-ci se définit moins par l'attachement aux valeurs démocratiques que par une pulsion conquérante et un désir d'éradiquer l'altérité (50). Le constat de Délivrance est sans appel: cette fois «la guerre sauvage ne produit pas l'homme blanc supérieur étatsunien, mais un homme dégénéré, altéré et démasculinisé » (56).

5 Le deuxième chapitre, «Dirty Harry: le retour du tueur d'Indiens ", évoque un cinéma où l'usage de la violence est affirmé comme étant nécessaire afin de maintenir l'ordre ou, plus exactement, de produire un ordre nouveau. Selon l'auteur, le film de Don 
Siegel se situe dans la directe filiation du mythe de la Frontière, réaffirmant «la croyance rooseveltienne [selon laquelle] la violence contre le sauvage revigore une civilisation déclinante » (78). Dans ce western urbain, l'inspecteur Harry est le digne héritier de John Wayne « aux méthodes douteuses mais à la morale inflexible » (81), un justicier à la violence rédemptrice.

6 Le troisième chapitre, "Platoon : la civilisation face aux barbares ", développe l'analyse de fictions vietnamistes esquissée au début du chapitre précédent qui s'appuyait sur le processus narratif visant à déculpabiliser le héros violent. La violence du colonisateur et de la civilisation n'est jamais présentée comme une agression mais comme une légitime défense : c'est l'environnement non civilisé auquel est confronté le héros qui s'affirme comme "la raison première de l'ensauvagement des soldats", tandis que «l'accent est mis sur le traumatisme individuel aux dépens de la responsabilité collective " (76). Le mythe de la Frontière revêt ici une fonction légitimante afin de justifier l'intervention étatsunienne au Vietnam et de donner un sens aux événements historiques tragiques. Citant Dittmar et Michaud, l'auteur démontre que «le Vietnam [cinématographique] est présenté comme quelque chose qui est arrivé, pas comme quelque chose que l'on a fait » (77). Et en privilégiant le point de vue du simple soldat, des récits filmiques comme Platoon ou Rambo permettent de concentrer l'attention sur le « chaos du combat » tout en évitant d'aborder les motivations politiques du conflit. La guerre est naturalisée, la faute historique de la défaite effacée, tandis que l'héroïsme mythique du guerrier étatsunien s'en trouve renforcé (115).

7 La deuxième partie, qui s'ouvre avec le chapitre 4 «Planet of the Apes: la chute de l'empire étatsunien", est consacrée au contre-mythe qui se pose en critique de l'expérience coloniale étatsunienne. Si cette dernière est alors réinterprétée comme une "agression tragique», l'auteur précise bien que le contre-mythe maintient néanmoins l'homme blanc au centre du nouveau récit. Il situe l'émergence de la critique de l'esprit de conquête dans le cinéma de science-fiction dystopique à partir de La Planète des singes qu'il qualifie de premier film de science-fiction à emprunter les formes et motifs du western, les héros perpétuant la conquête étatsunienne en la déplaçant vers d'autres horizons (160-161). L'empire simiesque y est en effet marqué des mêmes inégalités raciales, les cavaliers gorilles incarnant par exemple les cowboys et s'inscrivant dans la lignée de l'impérialisme étatsunien (176). Mais si le film de Franklin Schaffner dénonce bien la hiérarchie établie entre sauvagerie et civilisation, tout en ébranlant les certitudes idéologiques du mythe de la Frontière (« le racialisme, le progressisme, l'esprit de conquête et la violence régénératrice qui caractérisent le mythe rooseveltien [...] explosent avec la conscience que la civilisation contient en germe sa propre destruction » [177]), il ne donne en revanche aucune explication quant aux origines de ce racisme et, naturalisant les inégalités, il échoue selon l'auteur à considérer la race comme une construction sociale (171).

8 La même analyse se poursuit dans le chapitre suivant consacré à "Easy Rider: l'Amérique indianisée ", le film de Dennis Hopper s'imposant tout autant comme un hommage à la conquête que comme sa critique. S'il mobilise les caractéristiques du western, c'est certes pour mieux s'en distinguer mais il n'en remet pas foncièrement en cause la symbolique pour autant (202). Ainsi le développement narratif du road movie ne met pas réellement fin à la conquête mais montre plutôt une façon de «conquérir autrement » (199). Et si le contre-mythe dont il se réclame lui permet de remettre en cause la violence de l'impérialisme étatsunien, son éradication de l'altérité et son 
maintien d'une certaine hiérarchie raciale le placent néanmoins dans la pure tradition rooseveltienne - comme c'est d'ailleurs le cas pour bon nombre d'autres films reposant sur le schéma du héros blanc indianisé (206).

9 Le dernier chapitre, intitulé "Star Wars: le retour de l'Amérique missionnaire", développe la figure précédemment esquissée du héros blanc «ami des Indiens dont il pleure la disparition » et dont le "plus grand ennemi est l'empire étatsunien » (233), rétablissant l'Amérique dans son rôle de défenseure de la liberté aux côtés des populations insurgées. Et tandis que La Planète des singes, dans un climat post-bombe atomique, a marqué la science-fiction par sa remise en cause des bienfaits de la technologie et du progressisme, George Lucas a précisément conçu Star Wars IV: Un nouvel espoir comme un remède au pessimisme ambiant en choisissant de l'imprégner du romantisme et de la dimension épique de la conquête (237). Appartenant au genre $\mathrm{du}$ space opera qui est, selon l'auteur, la branche de la science-fiction la plus directement influencée par le mythe de la Frontière (235), Star Wars IV est structurellement organisé comme un récit turnerien et racialement construit comme un récit rooseveltien (241). Les êtres humains y forment les groupes dominants et parlent anglais, les robots ne sont que leurs serviteurs tandis que les extraterrestres subalternes parlent un zoulou, swahili ou quechua modifiés ou montés en version accélérée. Comme c'était déjà le cas avec La Planète des singes, l'univers star warsien reproduit les hiérarchies historiques des empires coloniaux dans une "relative dépolitisation et déhistoricisation du contexte induite par la science-fiction » qui ne font que naturaliser les inégalités et "présenter un monde impérial comme un récit universel » (244). De même, la posture apolitique d'une saga comme celle d'Indiana Jones et son « traitement comique de certains stéréotypes classiques ne font que renforcer la naturalité d'un univers où les minorités sont minorisées » (257).

10 Tout au long de Guerre sauvage \& empire de la liberté, Hervé Mayer livre une analyse convaincante des «modifications sémantiques et invariants syntaxiques » du mythe de la Frontière dans le cinéma étatsunien (238). Il identifie clairement deux versions de l'idéologie impériale qu'il fait correspondre au mythe et au contre-mythe. Dans les deux cas, l'homme blanc domine les autres mais c'est l'usage qu'il fait de cette supériorité qui diffère : là où l'Empire considère sa supériorité comme un mandat pour massacrer celles et ceux qui incarnent l'altérité, la rébellion considère quant à elle cette même supériorité comme une injonction à les protéger (248). La force de cette approche est de montrer que, même dans le cas de productions présentées comme de simples divertissements dépolitisés (une «bouffée d'air frais » dans le cas de Star Wars), tout film demeure inévitablement un objet politique et ne saurait se limiter au seul monde clos du cinéma. Au contraire, même le contre-mythe de la Frontière se retrouve miné par des structures narratives qui ne font que reproduire l'idéologie impériale qu'il vient pourtant critiquer (259).

11 Il convient ici de noter que l'analyse efficace de l'auteur s'appuie sur de nombreux exemples filmiques qui s'étendent bien au-delà des six œuvres principales composant son corpus primaire - ce que confirme la filmographie très fournie en fin d'ouvrage. Le choix éditorial d'identifier une œuvre principale par titre de chapitre mérite cependant d'être questionné, tant il réduit au premier abord la richesse du corpus analysé, mais aussi parce qu'il lui arrive de brouiller le propos réellement développé qui se trouve parfois en décalage avec ce que le titre semblait annoncer (le chapitre "Dirty Harry " commence par un détour via des films vietnamistes ; le film Rambo est largement étudié 
dans le chapitre "Platoon" et appuie tout autant le cœur de l'analyse qui y est développée ; les sagas Indiana Jones, Transformers et Star Trek occupent une bonne partie du chapitre «Star Wars ", etc.). Les intitulés plus généralistes choisis pour les chapitres de la thèse dont s'inspire l'ouvrage pouvaient sembler de ce point de vue plus pertinents. Par ailleurs, les titres des nombreuses sous-divisions qui structurent les chapitres et sous-parties sont parfois redondants, déclinant de manière peu distinctive les mêmes termes de violence, mythe/contre-mythe, Frontière, guerre, empire, etc. Les formulations choisies ne sont pas toujours assez spécifiques pour être signifiantes et l'effet de répétition dessert alors la fluidité et la pertinence de l'argumentation. Certains développements auraient sûrement gagné à être plus synthétiques et le sentiment de «déjà-lu » qui ponctue parfois la lecture laisse penser qu'un découpage par grandes thématiques transversales aurait peut-être été plus appropriée que la structure adoptée associant un chapitre à un film principal en particulier.

12 Autre élément rendant la lecture moins fluide, la traduction française des titres originaux des films n'est jamais fournie, que ce soit au fil du texte, en note de bas de page ou par la filmographie en fin d'ouvrage. Le livre étant publié en français, les films en question ayant été distribués en France et étant pour la plupart très connus sous leur titre traduit, ce parti-pris éditorial interroge, d'autant qu'aucune précision d'intention n'est fournie et que certaines notes viennent expliciter des éléments de contexte politico-historique étatsunien, laissant penser que l'ouvrage s'adresse à un lectorat plus large que les seules spécialistes en civilisation étatsunienne. La façon dont les citations sont traduites interroge également: dans la grande majorité des cas, elles sont en anglais dans le corps du texte et ensuite traduites en notes de bas de page, mais certaines se retrouvent directement intégrées au texte dans leur version française et la version originale est alors manquante. Ce parti-pris éditorial aurait lui aussi mérité d'être explicité, tandis qu'un manuscrit entièrement en français, avec les citations originales simplement reportées en notes, aurait incontestablement gagné en fluidité.

Les illustrations, nombreuses, se révèlent très utiles pour suivre l'argumentation détaillée de certaines séquences de films étudiées, d'autant que la mise en page associant aussitôt le photogramme à l'analyse correspondante en facilite l'appréhension. On ne peut que regretter la faible qualité des images qui manquent de contraste et de netteté, ce qui est d'autant plus surprenant que l'ouvrage bénéficie par ailleurs d'une très belle édition au rendu agréable (graphisme, typographie, choix du papier, etc.).

Mais le véritable point de faiblesse de l'ouvrage est ailleurs: le recours au terme "indigène " se révèle en effet problématique, d'autant qu'il est extrêmement récurrent. Or si d'autres concepts clés de l'argumentation ont été bien définis en introduction, ce n'est pas le cas pour "indigène » dont l'utilisation aurait pourtant méritée d'être mieux cadrée et les raisons la motivant, mieux explicitées. Il est tantôt synonyme d'Amérindien, d'Indien ou d'Autochtone (il est fait référence aux " populations indigènes » et au " territoire indigène » dans le contexte historique de la conquête de l'Ouest [22], le Blanc indianisé est le «dépositaire de l'esprit indigène quand l'Indien a disparu» [23], George Lucas se serait inspiré de "croyances amérindiennes " afin d'insuffler un peu de "sagesse indigène » à Star Wars [251]), tantôt synonyme de Vietcong, voire même de Japonais (dans le cas du Dernier samouraï [106]), d'Egyptien ou de Turc (dans le cas d'Indiana Jones traquant les «indigènes aux marges du monde développé » [256]). Le même terme semble à la fois renvoyer au seul 
contexte de la colonisation territoriale étatsunienne (« destruction de l'indigène par le colonisateur » [23]) et le dépasser largement, quand il ne désigne pas de façon globale l'Autre non occidental, non-civilisé, voire primitif (« violence contre l'indigène au nom de la civilisation" [24], "effacer l'indigène pour installer la civilisation" [75], «légitime défense de la civilisation contre les indigènes " [106]). Toutes ces réalités sont pourtant bien différentes, les contextes et signifiés non superposables. Il arrive souvent qu'on ait un peu de mal à identifier précisément ce à quoi l'« indigène » en question renvoie, ce qui peut rendre les propos de l'auteur confus.

La confusion est plus forte encore lorsque l'auteur évoque la «société autochtone de Deliverance ", faisant en réalité référence à la population rurale (blanche) locale de Caroline du Nord rencontrée par les personnages principaux originaires d'Atlanta (48). Des personnages « autochtones » qui sont de nouveau qualifiés d'« indigènes » quand est évoqué, un peu plus loin, le meurtre de l'un d'entre eux dont le corps se retrouve enterré au fond du lac artificiel (50). On lit par ailleurs, au sujet de Platoon, que «la réduction de l'ennemi au sauvage mythique racialise les Vietnamiens et déshumanise l'ensemble des indigènes, même les civils innocents " (126), tandis que dans La Planète des singes « le corps blanc d'Heston (...) subit toutes sortes de tortures et humiliations historiquement infligées aux indigènes et aux esclaves " (168). "Indigénisme » et « indianisme » semblent être utilisés de façon interchangeable, tandis qu'à partir du cinquième chapitre, "Easy Rider: l'Amérique indianisée ", le terme d'" Amérindiens " apparaît plus fréquemment sans que ce qui le distingue de l'indigène ou de l'Indien des chapitres précédents soit pour autant précisé. Tout au long de l'ouvrage, la confusion vient surtout du fait que nous ne sommes pas toujours en mesure de distinguer l'« indigène » en tant que figure filmique - incarnant l'Autre sauvage et étant de l'ordre des représentations stéréotypées qu'il s'agit précisément de déconstruire par l'analyse du mythe de la Frontière - de l'« indigène » en tant que membre de peuples bien réels en prise avec le colonialisme étatsunien ou un impérialisme globalisé. Il aurait été plus judicieux d'avoir recours à des termes plus spécifiques permettant de bien distinguer les deux situations considérées (réelle d'un côté, conceptuelle ou figurée de l'autre).

D'autant qu'historiquement, en français, le terme «indigène» possède une signification bien particulière qui nécessite d'interroger - ou du moins de préciser l'intentionnalité derrière son usage contemporain. Sous le régime de l'indigénat français (1881-1946), il renvoyait en effet à une catégorie officielle désignant les droits, les coutumes et les institutions particulières en vigueur pour les peuples colonisés de l'Empire. En Algérie par exemple, les citoyen nes de confession musulmane qui ne voulaient pas être régies par le droit civil français continuaient d'être régies par un droit, des institutions et un statut désignés comme "indigènes ». Ces sujets français étaient alors privés de la majeure partie de leurs droits et libertés, notamment la liberté d'aller et de venir ainsi que leurs droits politiques, le droit de vote et d'éligibilité. Par ailleurs, et ce à travers tout l'Empire, le terme "indigène " étant souvent utilisé par les colons pour désigner les habitantes des colonies considérées comme inférieur'es, il s'est inévitablement teinté d'une connotation négative et est progressivement devenu l'équivalent de «barbare » ou de "sauvage », désignant des individus non civilisés.

Depuis 1986, l'Académie française spécifie que le terme d'« indigène » est relatif aux « populations autochtones d'un pays sous régime colonial ou de protectorat / [à toute] personne qui appartient à la population autochtone d'un pays colonisé ». Il implique un 
rapport de domination et c'est précisément l'une des raisons pour lesquelles, dans le cadre du Groupe de travail sur la Déclaration des droits des peuples autochtones qui s'est réuni de 1987 à 2007 aux Nations unies, les représentantes autochtones francophones ont rejeté le terme pour la rédaction de textes officiels, souhaitant ainsi marquer une rupture avec la période de la colonisation et affirmer leur souveraineté. Le choix s'est porté sur le terme "autochtone" pour des raisons profondément politiques, appuyées par l'étymologie dont la distinction est précisée par le Littré : "Autochthone [du grec aûtos, "même", et khthôn, "terre"], qui est de la terre même ; Indigène, qui est né dans le pays ; Indigène [du latin indigena, "né dedans"] indique seulement les gens nés dans un pays; idée à laquelle autochthone ajoute que le peuple dont il s'agit a été de tout temps dans le pays et n'y est pas venu par immigration. Les créoles sont indigènes des Antilles; mais ils ne sont pas autochthones ». Les deux termes ne sont donc pas synonymes et se revendiquer "autochtone " permet aux membres de ces peuples - qui ont subi et, pour certains, subissent encore le colonialisme et diverses politiques assimilationnistes - d'affirmer leur présence sur le territoire préalablement aux vagues de colonisation. Continuer de les appeler " indigènes ", outre la connotation colonialiste du terme et le rapport de domination qu'il induit en contexte francophone, équivaut donc à leur refuser ce statut d'Autochtones qu'ils revendiquent, niant les combats liés au respect de leurs droits garantis par l'antériorité de leur occupation du territoire ${ }^{1}$.

Dans Guerre sauvage \& empire de la liberté, "autochtone " n'est (à de rares exceptions près) pas utilisé et le mouvement politique d'autodétermination auquel il renvoie (et dans lequel s'inscrivent pourtant les nations amérindiennes des États-Unis dont il est question dans l'ouvrage) n'est pas du tout évoqué. Il est par ailleurs regrettable que dans son exploration de la Frontière, l'auteur traite toutes les figures de l'altérité et de la sauvagerie comme étant quelque peu interchangeables. Son étude se situant en contexte étatsunien, il aurait été particulièrement intéressant de mentionner la spécificité des peuples amérindiens, leur antériorité territoriale faisant endosser un rôle spécifique à la figure de l'Indien au cinéma. Directement liée à l'émergence originelle du mythe de la Frontière, la disparition de l'Indien dans les récits filmiques ne revêt pas tout à fait la même signification que celle des autres ennemis raciaux incarnés dans le contre-mythe par les figures cinématographiques modernes de l'Arabe et du Vietcong. Il en est de même pour les concepts de colonisation et d'impérialisme qui, s'ils partagent de nombreux points communs, n'en sont pas pour autant synonymes. Ils auraient eux aussi mérité d'être mieux définis afin d'explorer leur éventuel traitement différentiel par le mythe et le contre-mythe de la Frontière notamment dans ce que le contexte colonial peut avoir de spécifique, en comparaison avec la culture globalisée au sein de laquelle opère l'impérialisme occidental.

Ces quelques limites mises à part, Guerre sauvage \& empire de la liberté n'en demeure pas moins une contribution très intéressante en ce qu'il dissocie de manière efficace le western et l'Ouest étatsunien historique du mythe de la Frontière (ou de son contremythe) qui s'impose comme le «cadre idéologique de référence du cinéma populaire étatsunien ». Loin d'avoir disparu lors du déclin du western, et bien plus vaste que lui dans ses possibles déclinaisons narratives, le mythe de la Frontière « reste au cœur de la formulation des problématiques du national et de son rapport à l'altérité dans le champ de la culture de masse » (273). Outre le nombre conséquent de films étudiés, il convient également de saluer la grande richesse des sources consultées - notamment les différentes versions de scénarios qui permettent à l'auteur de comparer et de 
révéler les intentions des cinéastes et autres responsables de production, ainsi que les raisons ayant motivé leurs variations. Cette incursion dans les coulisses de la création rend le récit d'autant plus passionnant, donnant immédiatement envie de se replonger dans chacun des classiques du cinéma étatsunien cités afin d'y observer, d'un œil dorénavant plus aiguisé, le mythe de la Frontière à l'œuvre.

\section{NOTES}

1. Voir notamment : BELLIER Irène, "L'Anthropologie, l'indigène et les peuples autochtones ", 19ème Conférence Robert Hertz à l'invitation de l'Association pour la Recherche en Anthropologie Social (APRAS), Juin 2011, Paris, France. halshs-00702527 [Consulté le 12/06/21] ; BELLIER Irène, "Autochtone.", EspacesTemps.net [En ligne], Dans l'air, 2009 [Consulté le 12/06/21] ; GERGAUD Sophie, Cinéastes autochtones, la souveraineté culturelle en action, Laval : Warm, 2019, 24-26.

\section{AUTEURS}

\section{SOPHIE GERGAUD}

Ethno-cinéaste indépendante 